\title{
Cavity QED Detection of Interfering Matter Waves
}

\author{
T. Bourdel, T. Donner, S. Ritter, A. Öttl, M. Köhl ${ }^{\dagger}$, and T. Esslinger \\ Institute of Quantum Electronics, ETH Zürich, Hönggerberg, CH-8093 Zürich, Switzerland
}

(Dated: July 3, 2018)

\begin{abstract}
We observe the build-up of a matter wave interference pattern from single atom detection events in a double-slit experiment. The interference arises from two overlapping atom laser beams extracted from a Rubidium Bose-Einstein condensate. Our detector is a high-finesse optical cavity which realizes the quantum measurement of the presence of an atom and thereby projects delocalized atoms into a state with zero or one atom in the resonator. The experiment reveals simultaneously the granular and the wave nature of matter.
\end{abstract}

PACS numbers: 03.75.Pp, 42.50.Pq, 05.30.Jp, 07.77.Gx

The prediction of the duality between particles and waves by de Broglie [1] is a cornerstone of quantum mechanics. Pioneering experiments have observed interferences of massive particles using electrons [2, 3], neutrons [4], atoms [5, 6, 7] and even large molecules [8]. However, the simple picture that matter waves show interferences just like classical waves neglects the granularity of matter. This analogy is valid only if the detector is classical and integrates the signal in such a way that the result is a mean particle flux. With quantum detectors that are sensitive to individual particles the discreteness of matter has to be considered. The probability to detect a particle is proportional to the square amplitude of the wave function and interferences are visible only after the signal is averaged over many particles.

In the regime of atom optics, single atom detection has been achieved for example by fluorescence [9], using a micro-channel plate detector for metastable atoms [10], and high-finesse optical cavities [1]. In these experiments the size of the de Broglie wave packet of the particles was much smaller than the detector area. Therefore localization effects during the detection have been negligible. With the realization of Bose-Einstein condensation in dilute gases particles with a wave-function of macroscopic dimensions have become experimentally accessible. Only very recently the single atom detection capability has been achieved together with quantum degenerate samples reaching the regime of quantum atom optics 12, 13]. The quantum nature of the measurement opens perspectives for atom interferometry at and below the standard limit [14].

For atoms with a spatially extended wave function, such as in a Bose-Einstein condensate or in an atom laser beam, a measurement projects the delocalized atom into a state localized at the detector [18]. This quantum measurement requires dissipation in the detection process. For single atom detection we employ a high-finesse optical cavity in the strong coupling regime of cavity quantum electrodynamics (QED) 15, 16, 17. We study this open quantum system including the two sources of dissipation, cavity losses and spontaneous emission. In particular, we calculate the time needed for the localisa- tion of an atom in the cavity measurement process. We then experimentally investigate atomic interferences on the single atom level using our detector.

A schematic of our experimental setup is shown in figure 1. We output couple two weak atom laser beams from a Bose-Einstein condensate and their wave functions overlap and interfere [19]. The flux is adjusted in such a way that there is on average only one atom at a time in the interferometer. Using the high-finesse cavity we measure single atom arrival times in the overlapping beams. We observe the gradual appearance of a matter wave interference pattern as more and more detection events are accumulated.

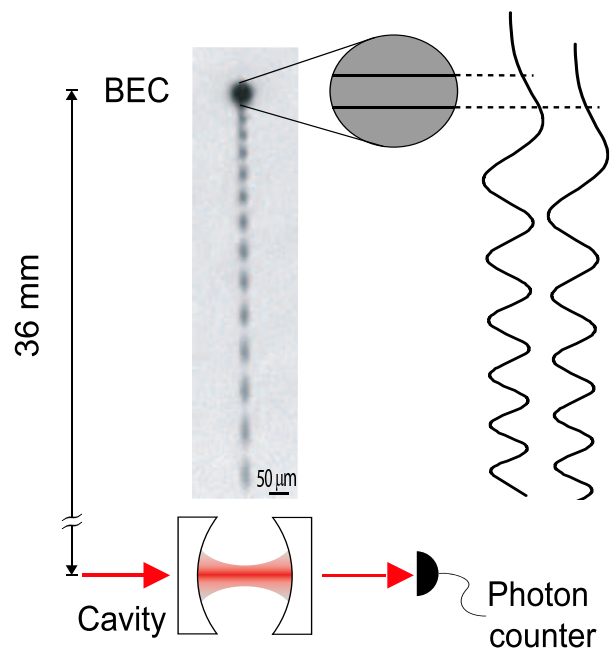

FIG. 1: Schematic of the experimental setup. From two well defined regions in a Bose-Einstein condensate (BEC), we couple atoms to an untrapped state. The real parts of the resulting atom laser wave-functions are sketched on the right hand side. The absorption image shows an interference pattern corresponding to $\Delta f=1 \mathrm{kHz}$ and to a flux $\sim 10^{6}$ times larger than in the actual single atom interference experiment. Monitoring the transmission through a high-finesse optical cavity with a photon counter, single atom transits are detected.

Single atom detection in an optical cavity can be captured in a classical picture: an atom changes the index of refraction in the cavity and thereby shifts it out of res- 

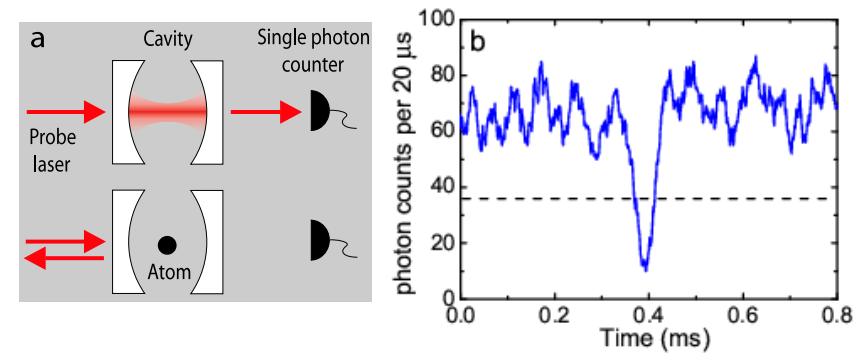

FIG. 2: a: Cavity single atom detection principle. An atom detunes the high-finesse cavity from resonance and the cavity transmission consequently drops. b: Photon flux through the high-finesse optical cavity when an atom is detected. The photon count rate is averaged over $20 \mu \mathrm{s}$. The detection threshold is set to be 4 times the standard deviation of the photon shot noise (dashed line).

onance from the probe laser frequency. In the absence of an atom, the probe beam is resonant with the cavity and its transmission is maximal. Experimentally we use a probe power corresponding to five photons on average in the cavity. The cavity lock is sufficiently stable for the cavity transmission to be at the photon shot noise limit. The presence of an atom results in a drop of the cavity transmission (see figure 2). We set the threshold for an atom detection event to a drop in transmission of four times the standard deviation of the photon shot noise in our $20 \mu$ s integration time. Then the overall detection efficiency of atoms extracted from a Bose-Einstein condensate is measured to be $0.23(8)$.

Our cavity has been described in a previous paper 12 and we only recall here its main figures of merit. Its length is $178 \mu \mathrm{m}$, the mode waist radius is $26 \mu \mathrm{m}$, and its finesse is $3 \times 10^{5}$. The maximum coupling strength between a single ${ }^{87} \mathrm{Rb}$ atom and the cavity field $g=$ $2 \pi \times 10.4 \mathrm{MHz}$ is larger than the cavity field decay rate $\kappa=2 \pi \times 1.4 \mathrm{MHz}$ and the atom spontaneous emission rate $\Gamma=2 \pi \times 6 \mathrm{MHz}$. The probe laser and the cavity are red-detuned as compared to the atomic resonance such that the light force pulls the atoms to regions where the coupling is large, therefore enhancing the detection efficiency.

To understand the actual detection process we study the dynamics of the atom-cavity quantum system taking into account dissipation. We first consider a classical atom entering a simplified square shaped cavity mode so that its coupling to the cavity field increases suddenly to a constant value $g$. The cavity field is initially coherent with a few photons. We use a two level approximation for the atom description and assume a $30 \mathrm{MHz}$ red-detuning of the probe laser compared to the atomic resonance [20]. In the case of strong coupling the following dynamics occur. On a short time scale given by $1 / g$, the atomcavity system exhibits coherent oscillations. It progressively reaches an equilibrium state on a time scale given by $1 / \kappa$ and $1 / \Gamma$ due to cavity loss and atomic spontaneous emission. These are the two sources of dissipation. In the equilibrium state, the mean photon number in the cavity is reduced and the cavity transmission drops.

To evaluate this drop quantitatively, we find the steady-state of the master equation for the density matrix numerically 21, 22, 23, 24]. For our parameters, the transmission as a function of the coupling strength $g$ is plotted in figure 3a. For a maximally coupled atom $g=2 \pi \times 10.4 \mathrm{MHz}$, the average intra-cavity photon number is found to be reduced from 5 to 0.9 , and the number of detected photons is then reduced by the same ratio. Such a reduction corresponds well to the largest observed transmission drops. An example is shown in figure 2. The detection threshold corresponds to a coupling of $g=2 \pi \times 6.5 \mathrm{MHz}$. Experimentally, unlike in our model, an atom feels a position dependent coupling as it transverses the mode profile. However the atom transit time through the cavity mode ( $40 \mu \mathrm{s})$ is long compared to the cavity relaxation time scales $1 / \kappa$ and $1 / \Gamma$ and the atomcavity system adiabatically follows a quasi equilibrium state. Therefore the experimental transmission drops can be compared to the calculated ones.

Specific to our experiment is that a spatially extended matter wave and not a classical atom enters the cavity. Our system allows us to realize a quantum measurement of the presence of an atom. For our low atom flux, we can neglect the probability of having more than one atom at a time in the cavity. The incoming continuous wavefunction is thus projected into a state with one or zero atom in the cavity. This projection necessarily involves decoherence that is introduced by spontaneous scattering and cavity photon loss. The origin of the decoherence can be understood as unread measurements in the environment [18, 21]. For example, if a spontaneously emitted photon is detected, there is necessarily an atom in the cavity and the wave-function is immediately projected. Similarly, the more different the light field with an atom in the cavity is from the field of an empty cavity, the more different is the scattered radiation out of the cavity, and the projection occurs correspondingly faster.

We now quantify the time needed for the projection to occur. For simplicity, rather than a continuous wave function, we consider a coherent mixture of one and zero atom entering a square shaped cavity at a given time. We take the limit when the probability to have one atom is low. The initial cavity field is the one of an empty cavity. Dissipation effects are studied by computing the time evolution of the density matrix [22]. The degree of projection of the initial state can be extracted from the off-diagonal terms between states with one atom and no atom in the density matrix. More precisely, we define the coherence as the square-root of the sum of the squared modulus of the off-diagonal terms mentioned above. This quantity is maximal for a pure quantum state with equal probability to have an atom or not. The coherence is 

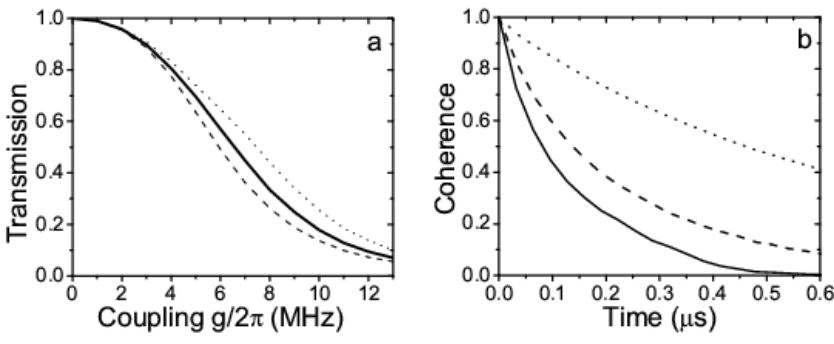

FIG. 3: a: Normalized transmission as a function of coupling strength. The solid line corresponds to our probe strength of 5 photons in the cavity in the absence of an atom. The dashed line is the weak probe limit. The dotted line corresponds to 10 photons in the cavity. b: Coherence between the states with one and no atom as a function of time. The initial coherence is normalized to 1 . Solid line: $g=2 \pi \times 10 \mathrm{MHz}$. Dashed line: $g=2 \pi \times 6.5 \mathrm{MHz}$. Dotted line: $g=2 \pi \times 3 \mathrm{MHz}$.

zero for a statistical mixture.

In figure $3 \mathrm{~b}$, the temporal evolution of the coherence is plotted. As expected, it decays to zero at long times due to dissipation. The decay time increases as the coupling to the cavity is weakened. In the limit where the coupling vanishes, the coherence is preserved. The atomic wave function then evolves as if there was no cavity. For $g>2 \pi \times 6.5 \mathrm{MHz}$, the decoherence time is found to be a fraction of a microsecond. This value is much lower than the $40 \mu$ s transit time of an atom through the cavity and for all our detected atomic transits, the wave function is thus well projected to a state with one atom. Our detection scheme realizes a quantum measurement of the presence of an atom in the cavity. However during an atom transit some photons are spontaneously scattered and the velocity of the atom is slightly modified.

Using our cavity detector, we can observe matter wave interferences on the single atom level. The starting point of the experiment is a quasi pure Bose-Einstein condensate with $1.5 \times 10^{6}$ Rubidium atoms in the hyperfine ground state $\left|F=1, m_{F}=-1\right\rangle[12]$. The atoms are magnetically trapped with frequencies $\omega_{\|}=2 \pi \times 7 \mathrm{~Hz}$ axially and $\omega_{\perp}=2 \pi \times 29 \mathrm{~Hz}$ radially. A weak and continuous microwave field locally spin-flips atoms from the BoseEinstein condensate into the untrapped $\left|F=2, m_{F}=0\right\rangle$ state. This process is resonant for a section of the condensate where the magnetic field is constant. Because the magnetic moment of the spin flipped atoms vanishes they fall due to gravity and form a continuous atom laser 25].

When we apply two microwave fields of different frequencies, we are able to output couple atom laser beams from two well defined regions of the condensate [19]. The two distinct atom laser wave functions overlap and interfere. At the entrance of the cavity, the atomic wave function $\psi$ is well described by the sum of two plane waves with the following time dependence

$$
\begin{aligned}
\psi(t) & \propto \exp \left(i \omega_{1} t\right)+\exp \left(i \omega_{2} t+\phi\right) \\
& \propto \cos \left(\left(\omega_{2}-\omega_{1}\right) t / 2+\phi\right)
\end{aligned}
$$

where $\hbar \omega_{1}$ and $\hbar \omega_{2}$ are the energies of the two laser beams and $\phi$ is a fixed phase difference. The radial dependence of the wave function is neglected. The probability to detect an atom is given by the square norm of the wave function which is modulated in time and behaves like a cosine squared. The modulation frequency of the interference signal is given by the energy difference between the two atom lasers. Experimentally, it is determined by the frequency difference of the two microwave fields and is chosen to be $\Delta f=10 \mathrm{~Hz}$, which corresponds to a distance of $5 \mathrm{~nm}$ between the two output coupling regions. The two microwave fields are generated such that the interference pattern is phase stable from one experimental run to the other.

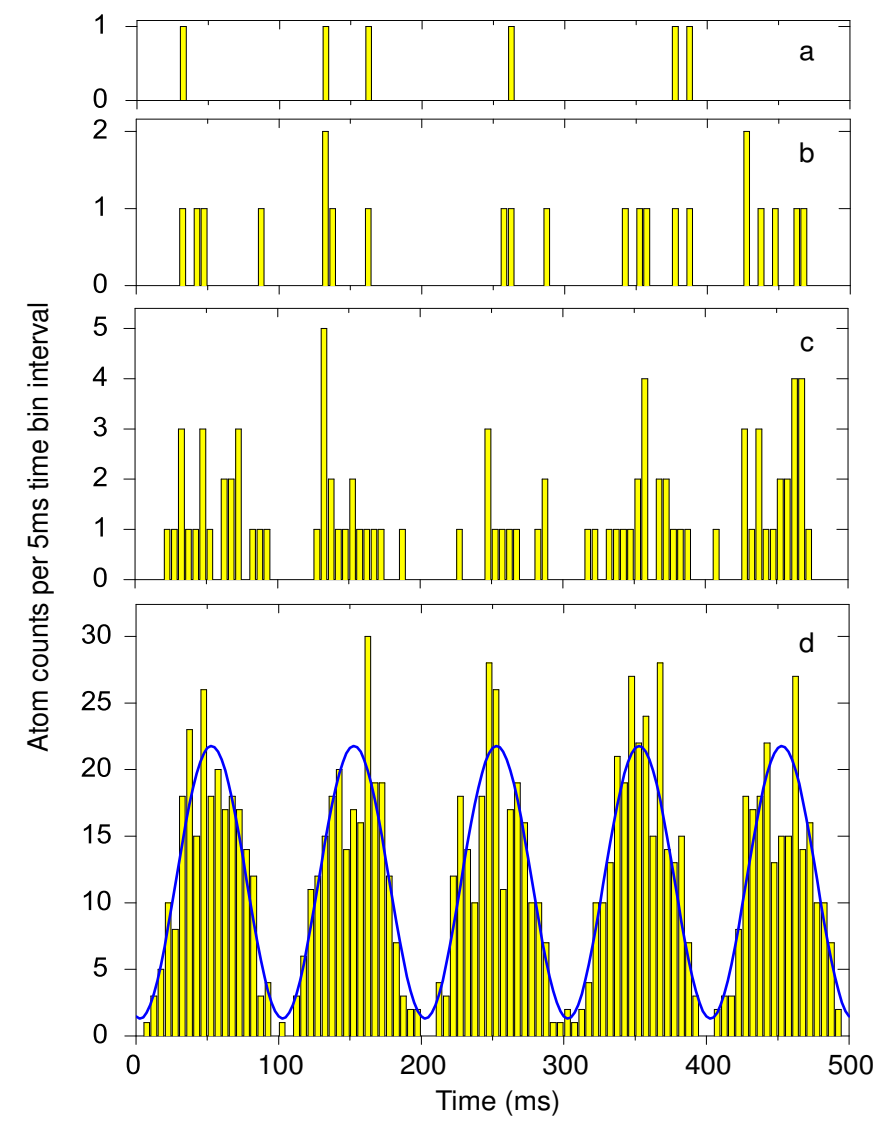

FIG. 4: Histograms of the atoms detected in $5 \mathrm{~ms}$ time bin intervals. a: Single experimental run. b: Sum of 4 runs. c: Sum of 16 runs. d: Sum of 191 runs, the line is a sinusoidal fit. Please note the different scales.

The results of the experiment are presented in figure 4. Each experimental run corresponds to output coupling from a new condensate. On average $\sim 6$ atoms are detected in $0.5 \mathrm{~s}$. After the detection of a few atoms, 
their arrival times appear to be random (fig. 4a). Nevertheless, after adding the results of several runs, an interference pattern progressively appears (fig. $4 \mathrm{~b}-\mathrm{d}$ ). The atom number fluctuation is found to be dominated by the atomic shot noise and the signal to noise ratio of the interference increases as more data are included. A fit to the histogram leads to a contrast of $0.89(5)$. The slight reduction of contrast is explained by a detected flux of about one atom every 3 runs in the absence of output coupling. We attribute this effect to artefact detection events and to atoms output coupled from stray magnetic fields.

We work with a flux of one detected atom per $83 \mathrm{~ms}$, which is about the time an atom needs to travel from the condensate region to the cavity. We are thus in a regime where the atoms fall one by one in the interferometer. A single atom behaves both like a wave because its time arrival probability shows an interference pattern and like a particle as single atoms are detected. This can be similarly expressed by saying that each individual atom is released from both slits simultaneously. Our experiment is an atomic counterpart of the Young's double slit experiment with individual photons.

To summarize, we detect matter wave interferences with a high-finesse optical cavity detector which realizes a quantum measurement of the presence of an atom. We explain how dissipation plays a crucial role in the detection process and for the localization of the atom inside the cavity. Using this detector, we are able to detect a high contrast atom interference pattern at the single atom level. The coupling of a matter wave to a cavity QED system opens the route to the quantum control not only of the internal state of the atoms but also of their positions [26]. Using the presented detection technique we can probe an atomic gas with a good quantum efficiency and introduce only a minimum perturbation through the measurement. This could facilitate non-destructive and time-resolved studies of the coherence of a quantum gas, for example during the formation of a Bose-Einstein condensate. Similar interference experiments between two distinct condensates would permit investigations of their relative phase evolution [27, 28] or diffusion [29].

We would like to thank G. Puebla for his help with the simulations. We acknowledge funding by SEP Information Sciences, OLAQUI (EU FP6-511057), Qudedis (ESF), and QSIT.

[†] Email: Koehl@phys.ethz.ch

[1] L. de Broglie, Nobel Lecture, 1929.
[2] C. Jönsson, Zeitschrift für Physik 161, 454 (1961).

[3] A. Tonomura, J. Endo, T. Matsuda, T. Kawasaki and H. Ezawa, American Journal of Physics 57, 117 (1989).

[4] A. Zeilinger, R. Gähler, C. G. Shull, W. Treimer, and W. Mampe, Rev. Mod. Phys. 60, 1067 (1988).

[5] O. Carnal and J. Mlynek, Phys. Rev. Lett. 66, 2689 (1991).

[6] D. W. Keith, C. R. Ekstrom, Q. A. Turchette, and D. E. Pritchard, Phys. Rev. Lett. 66 , 2693 (1991).

[7] F. Shimizu, K. Shimizu, and H. Takuma, Phys. Rev. A 46, R17 (1992).

[8] M. Arndt, O. Nairz, J. Vos-Andreae, C. Keller, G. Van der Zouw, A. Zeilinger, Nature 401, 1680 (1999).

[9] Z. Hu and H. J. Kimble, Optics Letters 19, 1888 (1994).

[10] J. Wiza, Nucl. Instr. and Meth. 162, 587 (1979).

[11] H. Mabuchi, Q. A. Turchette, M. S. Chapman, and H. J. Kimble, Opt. Lett. 21, 1393 (1996).

[12] A. Öttl, S. Ritter, M. Köhl, and T. Esslinger, Phys. Rev. Lett. 95, 090404 (2005).

[13] M. Schellekens, R. Hoppeler, A. Perrin, J. Viana Gomes, D. Boiron, A. Aspect, and C. I. Westbrook, Science 310, 648 (2005).

[14] P. Bouyer and M. A. Kasevich, Phys. Rev. A 56, R1083 (1997).

[15] C. J. Hood, M. S. Chapman, T. W. Lynn, and H. J. Kimble, Phys. Rev. Lett. 80, 4157 (1998).

[16] P. Münstermann, T. Fischer, P. Maunz, P. W. H. Pinkse, and G. Rempe, Phys. Rev. Lett. 82, 3791 (1999).

[17] J. A. Sauer, K. M. Fortier, M. S. Chang, C. D. Hamley, and M. S. Chapman, Phys. Rev. A 69, 051804(R) (2004).

[18] A. M. Herkommer, H. J. Carmichael, and W. P. Schleich, Quantum Semiclass. Opt. 8, 189 (1996).

[19] I. Bloch, T. W. Hänsch, and T. Esslinger, Nature 403, 166 (2000).

[20] Our probe beam is linearly polarized orthogonally to the magnetic field direction. We therefore do not probe on a cycling transition. The $30 \mathrm{MHz}$ detuning is an effective value chosen to match the experimental conditions.

[21] J. Dalibard, Y. Castin, and K. Mølmer, Phys. Rev. Lett. 68, 580 (1992).

[22] K. Mølmer, Y. Castin, and J. Dalibard, J. Opt. Soc. Am. B 10,524 (1993).

[23] C. Gardiner. Quantum Noise (Springer, Berlin, 1991).

[24] H. J. Carmichael, An Open system approach to Quantum Optics, Lecture Notes in Physics (Springer Verlag, Berlin, 1993).

[25] I. Bloch, T. W. Hänsch, and T. Esslinger, Phys. Rev. Lett. 82, 3008 (1999).

[26] P. Horak, S. M. Barnett, and H. Ritsch, Phys. Rev. A 61, 033609 (2000).

[27] M. Saba, T.A. Pasquini, C. Sanner, Y. Shin, W. Ketterle, and D.E. Pritchard, Science 307, 1945 (2005).

[28] M. Albiez, R. Gati, J. Fölling, S. Hunsmann, M. Cristiani, and M. K. Oberthaler, Phys. Rev. Lett. 95, 010402 (2005).

[29] J. Javanainen and M. Wilkens, Phys. Rev. Lett. 78, 4675 (1997). 